\title{
Variations spatiotemporelles du régime de reproduction de Dicorynia guianensis Amshoff (Caesalpiniaceae) en forêt guyanaise
}

\author{
Henri Caron ${ }^{\mathrm{a} *}$, Cyril Dutech ${ }^{\mathrm{b}}$, Éric Bandou ${ }^{\mathrm{a}}$ \\ a Institut national de la recherche agronomique, station de recherches forestières, \\ laboratoire de génétique Silvolab, BP 709, 97387, Kourou, Guyane française \\ ${ }^{\mathrm{b}}$ Centre de coopération internationale en recherche agronomique pour le \\ développement, UR diversité et amélioration génétique (DivAG), BP 5035 \\ 34 032, Montpellier, France
}

\begin{abstract}
Mating system of a Guiana tropical forest tree, Dicorynia guianensis Amshoff (Caesalpiniaceae). The mating system of a tropical rain forest tree Dicorynia guianensis (Caesalpiniaceae) was studied using allozyme analyses and the multilocus mixed mating programme of Ritland. Open-pollinated progenies were collected in 1993 and 1995 on logged and control plots of a sylvicultural trial in Paracou (French Guiana). D. guianensis exhibited a high outcrossing rate $(\mathrm{tm})$ for the global population which was remarkably stable in spite of a high variation of the flowering rate between the 2 years. The individual outcrossing rates $(t i)$ varied, but significant relations were not found between $t m$ or $t i$ and the density of flowering. Heterogeneity of pollen clouds existed at the patch and individual level, but not at the population level. Spatial autocorrelation statistics were used to investigate spatial structures of pollen clouds: there were not any significant spatial correlation. Pollen dispersion distances of up to $190 \mathrm{~m}$ were detected with rare allozymes. There was no significant difference between outcrossing rate when computing separate estimates for trees located on logged plots and control plots. (c) Inra/Elsevier, Paris
\end{abstract}

Dicorynia guianensis / tropical forest / mating system / pollen flow / Guiana

Résumé - Le régime de reproduction de Dicorynia guianensis (caesalpiniaceae), arbre de la forêt guyanaise a été étudié à l'aide des marqueurs enzymatiques. Le matériel végétal a été collecté en 1993 et 1995, sur le dispositif sylvicole de Paracou. Le taux d'allofécondation moyen de la population est élevé, $t m=0,85$, stable d'une année à l'autre malgré une variation importante du taux de floraison. Le taux individuel varie entre les arbres mais aucune corrélation n'a pu être établie avec des facteurs spatiaux, densité de floraison ou distance aux plus proches voisins.

\footnotetext{
* Correspondance et tirés à part
} 
L'hétérogénéité des nuages polliniques est sensible au niveau des agrégats et des arbres individuels, échelle spatiale où se déploie l'activité des insectes pollinisateurs. Nous n'avons en évidence aucune corrélation spatiale entre compositions des nuages polliniques individuels. Des distances de migration du pollen de 190 mètres ont été mise en évidence grâce aux allèles rares. Aucune différence significative du régime de reproduction de $D$. guianensis n'a été trouvée entre parcelles traitées et parcelles témoin du dispositif. (c) Inra/Elsevier, Paris

\section{arbre tropical / allogamie / flux pollinique / Guyane}

\section{INTRODUCTION}

Les informations concernant le fonctionnement des écosystèmes forestiers tropicaux et notamment guyanais sont encore très fragmentaires. À titre d'exemple, Carapa procera (meliaceae) est à notre connaissance, le seul arbre de la forêt guyanaise dont l'étude in situ du régime de reproduction ait fait l'objet d'une publication (Doligez et Joly, 1997).

Le système de reproduction est un facteur important de la structure génétique des populations, chez les plantes. Les espèces arborées tropicales présentent une grande variété de systèmes de reproduction, de mécanismes de pollinisation et de structures génétiques des populations (Hamrick et Murawski, 1990).

Le régime de reproduction dominant chez les arbres de la forêt tropicale ombrophile est l'allogamie plus ou moins stricte, avec un taux d'allofécondation dans les populations naturelles, qui a tendance à être élevé : sur 22 espèces d'arbres tropicaux étudiées, moins du quart ont un taux d'allofécondation inférieur à 0,8 , et la moitié d'entre elles supportent de 5 à $15 \%$ d'autofécondation $(\mathrm{Mu}-$ rawski, 1995). L'autofécondation peut augmenter quand la densité de la population baisse, comme cela a été mis en évidence au niveau populationnel et individuel chez Cavanillesia platanifolia (Murawski et Hamrick, 1991).

Le taux d'allofécondation est influencé par le synchronisme de floraison entre reproducteurs de la population, par l'efficacité des agents pollinisateurs dans les échanges entre arbres, et par l'existence de mécanismes d'autoincompatibilité plus ou moins stricts ou d'avortements sélectifs très précoces des fruits issus d'autofécondation.

En forêts tropicales, où la diversité spécifique est élevée et où beaucoup d'espèces d'arbres se maintiennent à des densités faibles, la pollinisation est très majoritairement zoophile, aussi l'abondance et le comportement des pollinisateurs déterminent-ils autant que le cycle phénologique des arbres, la réalisation de la pollinisation. Les abeilles sont les espèces de pollinisateurs, les plus nombreuses dans la canopée (Bawa, 1990). Les distances de migration du pollen peuvent être importantes (en moyenne $388 \mathrm{~m}$ chez Platipodium elegans, pollinisé par des insectes. Hamrick et Murawski, 1990). Une hétérogénéité des nuages polliniques est souvent mise en évidence dans les études concernant les arbres forestiers tropicaux : elle peut s'expliquer par la phénologie, l'action des pollinisateurs ou par une structuration spatiale génétique des arbres reproducteurs.

À l'échelle locale, les populations d'arbres forestiers tropicaux ont tendance à être génétiquement faiblement structurées, probablement en raison de flux géniques importants (Hamrick et Loveless, 1989).Cependant chez D. guianensis, 
Marque (1996) a mis en évidence, à l'aide des marqueurs chloroplastiques (à transmission uniparentale maternelle), une forte structuration spatiale des arbres adultes à l'échelle de l'agrégat $(0,5 \mathrm{ha})$, qui reflète la faible distance moyenne de dissémination des graines.

L'exploitation sylvicole peut avoir comme conséquence une baisse de densité des adultes reproducteurs, et/ou par les perturbations du milieu forestier, induire une modification importante de l'action des pollinisateurs : une densité plus faible due à l'exploitation forestière, s'accompagne d'une baisse significative du taux d'allofécondation moyen de la population en comparaison avec une zone témoin, chez Shorea megistophylla (Murawski et al., 1994). En revanche, dans une étude conduite à Paracou, la différence de densité des arbres reproducteurs potentiels de Carapa procera, entre parcelles témoin et traitées, n'étant pas significative, Doligez et Joly (1997) font l'hypothèse d'un changement de comportement des pollinisateurs pour rendre compte de la baisse significative du taux d'allofécondation, sur les parcelles exploitées.

Compte tenu de la faible densité des arbres reproducteurs et de la distribution agrégative des adultes, $D$. guianensis pourrait être sensible à un isolement par la distance : dans cette hypothèse, le régime de reproduction et la composition des nuages polliniques devraient refléter les différences de densité des reproducteurs et les variations temporelles de la floraison. En éliminant les plus gros arbres, l'exploitation sylvicole augmente l'isolement des individus et pourrait en accentuer les conséquences sur la reproduction.

Dans cette étude du régime de reproduction de $D$. guianensis, nous avons comparé le taux d'allofécondation et les nuages polliniques à différentes échelles spatiales et essayé de déterminer si ils étaient corrélés à des paramètres spatiaux tels que la densité de floraison ou la distance au plus proche voisin. Nous avons ensuite vérifié si les traitements sylvicoles avaient provoqué des modifications sensibles du régime de reproduction.

\section{MATÉRIELS ET MÉTHODES}

Dicorynia guianensis est une espèce endémique du plateau des Guyanes (du Surinam à l'état Brésilien de l'Amapa). C'est un arbre de la canopée pouvant atteindre $45 \mathrm{~m}$. La densité moyenne des arbres de $\mathrm{dbh}>10 \mathrm{~cm}$ (diamètre mesuré à 1,30 $\mathrm{m}$ de hauteur), est à Paracou de 6,9 arbres/ha, distribués en agrégats, de 0,4 ha en moyenne et comptant de 11 à 43 individus. Les agrégats sont distants de 48 à 151 m (78 m en moyenne), (Cabrera et Gignoux 1989, Deschères 1991). À l'échelle de la population, la floraison est synchrone, et régulière par cycle de deux ans (Loubry, 1994). Les pollinisateurs ne sont pas connus, mais le syndrome floral suggère une pollinisation par des insectes de moyenne ou grande taille (hymènoptères). La production de pollen ( $\mathrm{dbh}$ min. $22 \mathrm{~cm}$ ) est plus précoce dans la vie de l'arbre que la production de graines (dbh min. $28 \mathrm{~cm}$ ) (obs. pers.). Celle-ci peut être très abondante (10 à 25000 par arbre) mais une forte proportion de gousses (44\%) est stérile (Loubry, 1995). Elles sont disséminées à faible distance par le vent, $30 \mathrm{~m}$ en moyenne $(5 \%$ au-delà de $50 \mathrm{~m})$. La germination est étalée dans le temps et les plantules ont besoin d'une ouverture dans le couvert forestier pour pouvoir se développer. (Forget, 1988). 


\subsection{L'échantillonnage}

Le dispositif expérimental de Paracou (500 ha), mis en place par le CiradForêt en 1986, comporte quatre sous-groupes géographiques de parcelles, correspondant à trois répétitions d'un traitement sylvicole à quatre niveaux d'intensité croissante (figure 1). Les arbres dont le dbh est supérieur à $10 \mathrm{~cm}$, sont identifiés, cartographiés et mesurés, sur 120 ha (Schmitt, 1989).

L'échantillonnage a été hiérarchisé à priori (sous-groupes géographiques, agrégats, et semenciers). Les graines sont récoltées au pied des arbres, en évitant les zones de recouvrement entre deux semenciers voisins. Dans chaque descendance, 9 à 63 plantules ont été analysées. De plus, 64 reproducteurs potentiels de la P16 ont été directement génotypés ( $83 \%$ de l'effectif).

Le suivi phénologique a été fait sur l'ensemble du dispositif pour les arbres de $\mathrm{dbh}>30 \mathrm{~cm}$ : un à deux passages ont été réalisés au moment de la floraison et de la fructification de $D$. guianensis.

\subsection{Les analyses enzymatiques}

Les extraits sont réalisés à partir de cotylédons, de feuilles, ou de cambium d'adulte, dans un tampon d'extraction mis au point pour les espèces tropicales (Liengsiri et al., 1990).

\begin{tabular}{lccc}
\hline Système enzymatique & $\begin{array}{c}\text { Structure } \\
\text { de l'enzyme }\end{array}$ & $\begin{array}{c}\text { Nom } \\
\text { du locus }\end{array}$ & $\begin{array}{c}\text { Nombre } \\
\text { d'allèles }\end{array}$ \\
\hline Isocitrate déshydrogénase EC1.1.1.42 & dimérique & IDH1 & 3 \\
Phosphoglucomutase EC2.7.5.1 & monomérique & PGM & 2 \\
Phosphoglucoisomérase EC5.3.1.9 & dimérique & PGI & 3 \\
\hline
\end{tabular}

\subsection{L'analyse des données}

Nous avons utilisé le logiciel de Ritland (1990), qui estime les taux d'allofécondation multilocus $(t m)$ et monolocus $(t s)$ de la population et des individus $(t i)$, infère les génotypes maternels, et calcule les fréquences alléliques du pollen. Par un test de $\chi^{2}$, nous avons vérifié la ségrégation mendélienne aux loci étudiés, et par la procédure du jacknife la robustesse des estimations multilocus du taux d'allofécondation (données non publiées).

Nous avons estimé le taux d'allofécondation moyen de la population à partir de l'indice de fixation déterminé sur la population de plantules $(n=92$, une plantule par semencier $), t=(1-F) /(1+F)$.

L'estimation des fréquences polliniques se fait en ôtant du pool de gamètes d'une descendance, la part maternelle pour laquelle on fait l'hypothèse d'une ségrégation mendélienne. Nous avons travaillé sur le pollen total.

L'analyse de la structuration par autocorrélations spatiales a été faite à l'aide des logiciels morfreq et boot (Kremer et al., non publié) : on a recherché l'existence d'une liaison entre l'allèle le plus fréquent de chaque locus, dans les nuages polliniques individuels, et la distance entre les arbres (classes de distance de $30 \mathrm{~m}$, entre 0 et $600 \mathrm{~m}$ ) 


\section{RÉSULTATS}

\subsection{Phénologie de la floraison}

En 1993, le pourcentage d'arbres fleuris s'élève à $84 \%(n=183)$, et $54 \%$ $(n=246)$ en 1995. Les tests du $\chi^{2}$ d'homogénéité comparant les parties nord et sud du dispositif, ne sont significatifs pour aucune des deux années $\left(\chi^{2}\right.$ calculé $\left.<0,0001, \mathrm{ddl}=1\right)$. La comparaison des intensités de floraison entre groupes géographiques ne fait pas ressortir de différence nette. Il n'existe pas non plus, de différence significative de taux de floraison entre agrégats voisins. Entre traitements sylvicoles, la proportion d'arbres fleuris ne varie pas significativement (en $1993 \chi^{2}$ calculé $=4,78$, et en $1995 \chi^{2}$ calculé $\left.=4,70\right)$ : nous avons seulement observé que les arbres peuvent fleurir un peu plus jeunes $(\mathrm{dbh}=22 \mathrm{~cm})$, sur les parcelles, où le traitement a été le plus intense.

\subsection{Variations du taux d'allofécondation}

Le taux d'allofécondation moyen de la population $(\mathrm{tm})$ à l'échelle du dispositif de Paracou (500 ha) est très stable d'une année à l'autre : on observe environ $15 \%$ d'autofécondation, malgré un taux de floraison différent entre $1993(84 \%)$ et 1995 (54\%). Les valeurs de $t m$ obtenues avec trois loci par la procédure du «jacknife», restent très voisines (données non publiées). L'estimation indirecte du $t m=0,830(F=0,093)$ n'est pas significativement différente de la valeur trouvée par le modèle $t m=0,854(0,03)$. Pour aucune des estimations, on n'observe de différence significative entre les estimations multilocus et monolocus.

En 1993, une différence non significative, existe entre les sous-groupes géographiques ( $80 \mathrm{ha}$ ), mais on remarque qu'elle est de même amplitude que celle observée entre les deux années, 1993 et 1995, à l'échelle de la parcelle 16 (tableau I).

Les estimations du taux d'allofécondation individuel $(t i)$ peuvent être très différentes pour un même arbre, selon qu'on impose au nuage pollinique fécondant chaque arbre, la composition moyenne estimée de la population ( $p$ fixé) ou une composition estimée individuellement pour chaque arbre ( $p$ non fixé) ; cette dernière option n'est pas recommandée quand le nombre de loci est faible (Ritland et El-Kassaby, 1985). Sur un effectif de dix-neuf arbres en 1993, et de sept arbres en 1995, elles sont comprises entre 0,48 et 1,35 la première année, et entre 0,44 et 0,94, la seconde. Les estimations sont significativement inférieures à $1(p<0,05)$, dans huit cas sur $19(42 \%)$ en 1993, et quatre cas sur six $(67 \%)$ en 1995 (tableau II).

La comparaison du taux d'allofécondation moyen, entre les parcelles traitées et les parcelles témoins, ne met en évidence aucune différence significative, ni de $t m$ ni de $(t m-t s)($ tableau $V)$.

Nous observons la stabilité et la robustesse des estimations des taux d'allofécondation à l'échelle de la population, et des différences significatives à l'échelle individuelle, ce qui a déjà été constaté dans de nombreux cas. 
Table I. Taux de floraison (en \%), estimations multilocus $(\mathrm{tm})$ et monolocus $(\mathrm{ts}) \mathrm{du}$ taux d'allofécondation moyen de la population à différentes échelles spatiales.

\begin{tabular}{|c|c|c|c|c|c|c|c|}
\hline Population & Surface & $\begin{array}{c}\text { Nombre } \\
\text { de } \\
\text { familles }\end{array}$ & $\begin{array}{c}\text { Nombre } \\
\text { de } \\
\text { plantules }\end{array}$ & $\begin{array}{c}\text { Floraison } \\
(\%)\end{array}$ & $t m$ & ts & $t m-t s$ \\
\hline Dispositif 1995 & 500 & 32 & 727 & 54,5 & $\begin{array}{c}0,846 \\
(0,057)\end{array}$ & $\begin{array}{c}0,861 \\
(0,071)\end{array}$ & $\begin{array}{c}-0,016 \\
(0,019)\end{array}$ \\
\hline Dispositif 1993 & 500 & 73 & 1624 & 84 & $\begin{array}{c}0,854 \\
(0,031)\end{array}$ & $\begin{array}{c}0,837 \\
(0,033)\end{array}$ & $\begin{array}{c}0,017 \\
(0,012)\end{array}$ \\
\hline NE 1993 & 80 & 12 & 216 & 77,4 & $\begin{array}{c}0,882 \\
(0,055)\end{array}$ & $\begin{array}{c}0,816 \\
(0,051)\end{array}$ & $\begin{array}{c}0,066 \\
(0,031)\end{array}$ \\
\hline NW 1993 & 80 & 12 & 213 & 93 & $\begin{array}{c}0,834 \\
(0,088)\end{array}$ & $\begin{array}{c}0,774 \\
(0,083)\end{array}$ & $\begin{array}{c}0,059 \\
(0,05)\end{array}$ \\
\hline SE 1993 & 80 & 14 & 271 & - & $\begin{array}{c}0,823 \\
(0,774)\end{array}$ & $\begin{array}{c}0,826 \\
(0,077)\end{array}$ & $\begin{array}{c}-0,003 \\
(0,014)\end{array}$ \\
\hline P16 1993 & 25 & 35 & 924 & 78,1 & $\begin{array}{c}0,891 \\
(0,042)\end{array}$ & $\begin{array}{c}0,906 \\
(0,044)\end{array}$ & $\begin{array}{c}-0,015 \\
(0,013)\end{array}$ \\
\hline P16 1995 & 25 & 19 & 455 & 64 & $\begin{array}{c}0,836 \\
(0,089)\end{array}$ & $\begin{array}{c}0,842 \\
(0,105)\end{array}$ & $\begin{array}{c}-0,066 \\
(0,25)\end{array}$ \\
\hline
\end{tabular}

\subsection{Variations des nuages polliniques}

Les tests $\chi^{2}$ de comparaison de la population d'ovules et de celles des grains de pollen, échantillonnées sur trois aires de taille différente, et sur deux années (tableau III), ne sont significatifs que dans trois cas sur douze : au locus pgm, $(p<0,05)$, en 1993 à l'échelle de 300 ha; et surtout au locus idh $2,(p<0,001)$, à l'échelle de 25 ha (P16) en 1993 et 1995. En ôtant de l'échantillon, l'arbre 16(14)1 qui présente une distorsion de ségrégation, le test n'est plus significatif en $1995\left(\chi^{2}=0,96,2 \mathrm{ddl}\right)$, et la valeur du $\chi^{2}$ baisse sensiblement en 1993 $\left(\chi^{2}=16,3,2 \mathrm{ddl}, p<0,001\right)$; d'autre part, en 1993, l'allèle 2, rare à ce locus, a de faibles effectifs, ce qui pose un problème de validité du test statistique (tableau III).

La comparaison interannuelle de la composition moyenne des nuages polliniques met en évidence une remarquable stabilité, tant à l'échelle du dispositif (500 ha) qu'à celle de la parcelle 16 (25 ha) (tableau IV). Les différences entre groupes géographiques ne se marquent que pour les allèles «rares», une fréquence plus élevée de l'allèle 1 de PGI pour NE, et de l'allèle 3 de IDH1 pour SE. A l'échelle de la parcelle 16, les variations de composition du nuage pollinique, entre les agrégats une année donnée, et interannuelles pour un même agrégat, sont de même amplitude. Cela reste vrai au niveau individuel, avec des amplitudes beaucoup plus grandes (figure 2).

Les corrélogrammes qui portent sur l'allèle le plus fréquent de chacun des quatre loci, ne met en évidence aucune relation significative entre composition des nuages polliniques individuels et distance entre les arbres (données non publiées). 
Table II. Taux d'allofécondation individuel ( $t i, p$ fixé et $p$ non fixé), et densité des arbres voisins de la même espèce sur la parcelle 16 (nombre d'arbres fleuris dans un rayon de $30 \mathrm{~m}$ (D1), $60 \mathrm{~m}$ (D2), $150 \mathrm{~m}$ (D3)).

\begin{tabular}{lccccccc}
\hline Individu & & $\begin{array}{c}\text { Nombre } \\
\text { de plantules }\end{array}$ & $\begin{array}{c}t i \\
p \text { fixé }\end{array}$ & $\begin{array}{c}t i \\
p \text { non fixé }\end{array}$ & D1 & D2 & D3 \\
\hline 1,261 & $(1995)$ & 51 & $0,46(0,08)$ & $0,44(, 08)$ & 5 & 6 & 19 \\
2,183 & $(1993)$ & 33 & $0,54(0,15)$ & $0,84(0,54)$ & 11 & 12 & 36 \\
2,187 & $(1993)$ & 24 & $0,59(0,19)$ & $0,48(0,24)$ & 11 & 12 & 36 \\
22,185 & $(1993)$ & 42 & $0,60(0,17)$ & $0,57(0,17)$ & 1 & 6 & $6^{*}$ \\
2,145 & $(1993)$ & 43 & $0,62(0,11)$ & $0,57(0,15)$ & 9 & 12 & $22^{*}$ \\
25,200 & $(1993)$ & 34 & $0,63(0,10)$ & $0,69(0,22)$ & 0 & $4^{*}$ & $8^{*}$ \\
22,228 & $(1995)$ & 47 & $0,64(0,07)$ & $0,72(0,15)$ & 1 & $1^{*}$ & $3^{*}$ \\
20,145 & $(1993)$ & 63 & $0,77(0,34)$ & $1,29(0,21)$ & 3 & 6 & $13^{*}$ \\
4,420 & $(1995)$ & 50 & $0,77(0,19)$ & $0,83(0,21)$ & 0 & 0 & $3^{*}$ \\
20,445 & $(1993)$ & 34 & $0,77(0,13)$ & $0,92(0,42)$ & 4 & $6^{*}$ & $10^{*}$ \\
14,1 & $(1995)$ & 61 & $0,83(@)$ & & 0 & 0 & 7 \\
14,1 & $(1993)$ & 54 & $0,85(@)$ & & 0 & 0 & 11 \\
2,34 & $(1993)$ & 49 & $0,86(0,10)$ & $0,66(0,10)$ & 5 & 12 & 36 \\
14,470 & $(1993)$ & 39 & $0,89(0,07)$ & $0,83(0,10)$ & 1 & 1 & $14^{*}$ \\
24,523 & $(1995)$ & 52 & $0,94(0,09)$ & $0,88(0,12)$ & 0 & 1 & $7^{*}$ \\
20,384 & $(1993)$ & 51 & $0,94(0,26)$ & $1,57(0,18)$ & 6 & 6 & $13^{*}$ \\
12,313 & $(1993)$ & 45 & $1,03(0,12)$ & $1,34(0,14)$ & 5 & 6 & 14 \\
22,463 & $(1993)$ & 52 & $1,13(0,21)$ & $1,07(0,09)$ & 3 & $4^{*}$ & $6^{*}$ \\
9,8 & $(1993)$ & 39 & $1,29(0,25)$ & $1,20(0,26)$ & 1 & 1 & 10 \\
2,163 & $(1993)$ & 49 & $1,35(0,24)$ & $1,58(0,20)$ & 11 & 12 & 36 \\
\hline
\end{tabular}

(@) : calcul direct = nombre hétérozygotes /nombre total, au locus idh1 (semencier inféré homozygote 3.3$) ;^{*}$ : nombre minimal de pères potentiels; effet de bord.

Table V. Comparaison des taux d'allofécondation dans les parcelles traitées et témoin du dispositif sylvicole de Paracou (1993).

\begin{tabular}{|c|c|c|c|c|c|}
\hline & $\mathrm{Nf}$ & $\mathrm{Np}$ & $t m$ (e.t) & $t s$ (e.t) & $t m-t s$ (e.t) \\
\hline Traitées & 15 & 272 & $\begin{array}{c}0,841 \\
(0,075)\end{array}$ & $\begin{array}{c}0,790 \\
(0,072)\end{array}$ & $\begin{array}{c}0,051 \\
(0,023)\end{array}$ \\
\hline Témoin & 23 & 427 & $\begin{array}{c}0,852 \\
(0,04)\end{array}$ & $\begin{array}{c}0,810 \\
(0,043)\end{array}$ & $\begin{array}{c}0,042 \\
(0,019)\end{array}$ \\
\hline
\end{tabular}

$\mathrm{Nf}$ : nombre de descendances analysées; $\mathrm{Np}$ : nombre total de plantules analysées.

\subsection{Flux de gènes}

Les allèles rares sur la P16, (1 de PGI, 3 de IDH1, 2 de IDH2) permettent de mettre en évidence des distances de pollinisation au moins égales à $190 \mathrm{~m}$, et des échanges polliniques dans les deux sens, entre les arbres de deux agrégats (carrés 7 et 9) distants de plus de $180 \mathrm{~m}$ (figure 1). Il faut cependant noter que 
Table III. Test $\chi^{2}$ d'homogénéité des fréquences alléliques du pollen et des ovules. $(65<$ nbre ovules $<77 ; 619<$ nbre gamètes mâles $<955)$.

\begin{tabular}{lcccc}
\hline pop/locus & PGI & PGM & IDH1 & IDH2 \\
\hline P16 93 (25 ha) & $\chi^{2}=\begin{array}{c}3,592 \mathrm{ddl} \\
\text { NS }\end{array}$ & $\begin{array}{c}\chi^{2}=3,651 \mathrm{ddl} \\
\text { NS }\end{array}$ & $\begin{array}{c}\chi^{2}=2,052 \mathrm{ddl} \\
\text { NS }\end{array}$ & $\begin{array}{c}\chi^{2}=30,3 \text { 3ddl } \\
p<0,001\end{array}$ \\
disp 93 (300 ha) $)$ & $\chi^{2}=\begin{array}{c}4,90 \\
\text { NS }\end{array}$ & $\chi^{2}=5,391 \mathrm{ddl}$ & $\chi^{2}=0,942 \mathrm{ddl}$ & $\chi^{2}=0,182 \mathrm{ddl}$ \\
& & $p<0,05$ & NS & NS \\
disp 95 (500 ha) & $\chi^{2}=2,02 \mathrm{ddl}$ & $\chi^{2}=1,411 \mathrm{ddl}$ & $\chi^{2}=1,912 \mathrm{ddl}$ & $\chi^{2}=12,13 \mathrm{ddl}$ \\
& NS & NS & NS & $p<0,01$ \\
\hline
\end{tabular}

Table IV. Variations interannuelles des fréquences alléliques du nuage pollinique (en \%) à l'échelle des agrégats de la P16. (Allèle le plus fréquent).

\begin{tabular}{lcccccc}
\hline Locus & Année & Dispositif & P16 & Ag.C1 & Ag.C2 & Ag.C22 \\
\hline $\begin{array}{l}\text { Nombre } \\
\text { plantules }\end{array}$ & 1993 & $1588(33)$ & $924(36)$ & $112(8)$ & $246(7)$ & $127(4)$ \\
(arbres) & 1995 & $764(33)$ & $497(20)$ & $96(4)$ & $73(3)$ & $87(3)$ \\
Pgi all 2 & 1993 & 49,8 & 47,2 & 57,6 & 35,8 & 60,2 \\
& 1995 & 49,7 & 46,6 & 69,9 & 45,8 & 29 \\
Pgm all2 & 1993 & 67,7 & 70,5 & 76,1 & 68,1 & 77 \\
& 1995 & 67,8 & 71,7 & 86,1 & 91,5 & 51,7 \\
Idh1 all2 & 1993 & 83,9 & 85 & 91,4 & 83,9 & 77,8 \\
& 1995 & 74,9 & 69,4 & 59,6 & 49,3 & 75,9 \\
Idh2 all1 & 1993 & 54,6 & 51,7 & 52,7 & 76,7 & 50,8 \\
& 1995 & 59,4 & 60 & 73,2 & 81,9 & 47,1 \\
\hline
\end{tabular}

les distances de migration du pollen autour du père «donneur » sont en rapport avec la distance moyenne qui le sépare de ses voisins immédiats : élevées quand l'arbre est isolé (cas du (14)1 ou du (9)226), faibles, quand il est dans un agrégat dense (carré 22 et 2 ). La fréquence très élevée $(>50 \%)$ en 1995, chez les trois arbres voisins (2) 145, (2)163 et (2)177, de l'allèle 3 de IDH1 dont les donneurs les plus probables sont (2)171, (2)301, (2)302, montre qu'une bonne partie du pollen peut venir des proches voisins (figure 3).

La plus grande similarité en 1993, des nuages polliniques pour les allèles les plus fréquents, de (2)145 et (2)163 qui sont directement voisins, par rapport à (2) 177 plus proche de (2)163 mais séparé de lui par d'autres $D$. guianensis, renforce cette hypothèse (figure 2 ).

\section{DISCUSSION}

La durée et le bon synchronisme de la floraison à l'échelle locale, confirmés par Loubry (1994), semble limiter fortement l'isolement reproductif temporel 


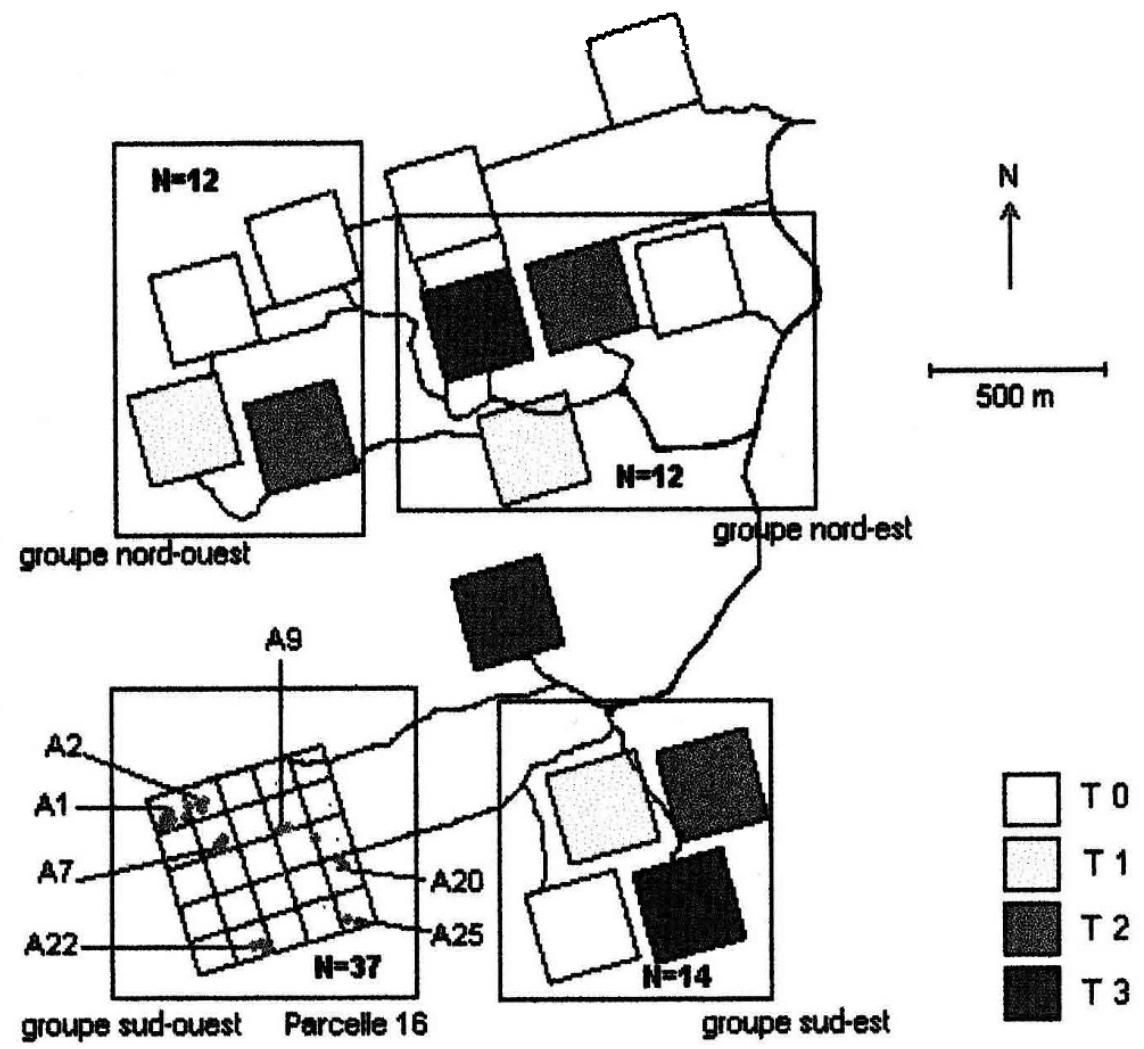

\section{(N : nombre de semenciers échantillonnés; Ai : aggrégat )}

Figure 1. Dispositif de Paracou (T0 : témoins ; T1, T2, T3 : traitements sylvicoles d'intensité croissante). ( $\mathrm{N}$ : nombre de semenciers échantillonnés; Ai : agrégat). Chaque arbre inventorié est identifié par un code, par exemple : 16(14)1: arbre $\mathrm{n}^{\circ} 1$ du carré 14 de la parcelle 16.

au cours d'une saison. En revanche, le comportement phénologique de la population pourrait expliquer en partie les variations interannuelles de la composition des nuages polliniques et des taux individuels d'allofécondation, si on prend en compte d'une part la variation interannuelle du taux de floraison de la population alors que la densité de reproducteurs est faible ( 3 arbres/ha), et d'autre part, les différences d'intensité de floraison entre les individus (observation personnelle).

D. guianensis confirme les résultats antérieurs concernant une forte prééminence de l'allogamie chez les arbres tropicaux, bien que ces espèces ont souvent de faibles densités de reproducteurs (Bawa 1992, Murawski, 1995); un taux moyen d'allofécondation comparable, a été trouvé chez des populations de Shorea congestiflora (Murawski et al., 1994), Stemonoporus oblongifolius, (Murawski et Bawa, 1994), Bertholettia excelsia (O. Malley et al., 1988). D. guianensis tolère un taux faible, mais significatif (de 10 à $15 \%$ ), d'autofécondation; puisque la différence $(t m-t s)$ n'est jamais significative, la distribution 

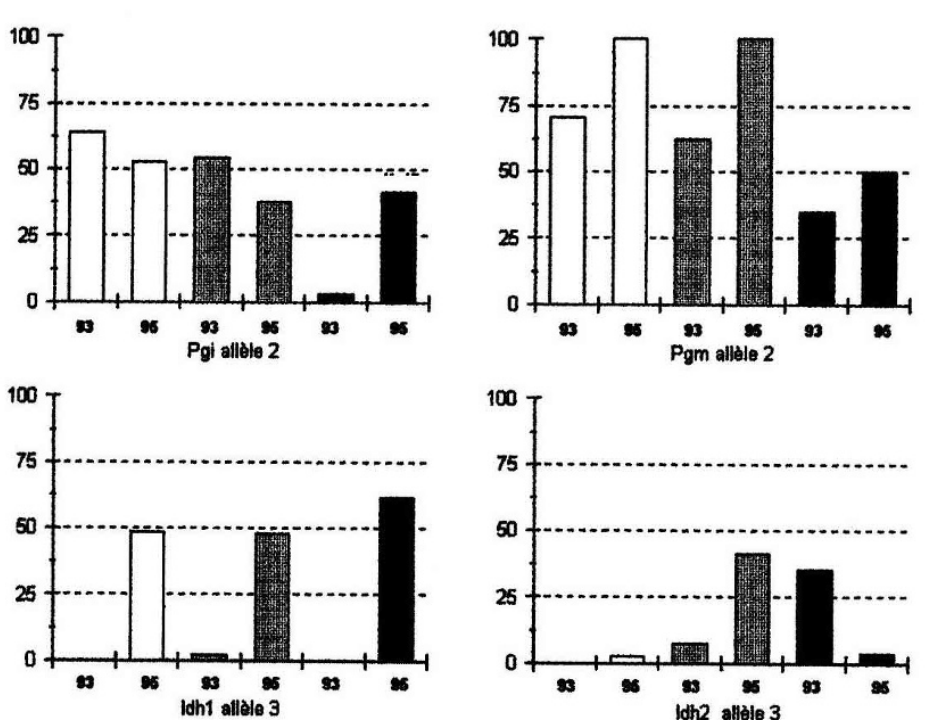

$\begin{array}{ccc}\text { (effectifs : } & 1993 & (2) 145: 43 \\ & 1995 & (2) 145: 36\end{array}$
(2) $163: 46$
(2) $177: 34$
(2) $163: 24$
(2) $177: 13$ )

Figure 2. Variations interannuelles des nuages polliniques individuels de trois arbres du carré 2 de la parcelle 16 .
Effectifs : 1993
(2) $145: 43$
(2) $163: 46$
(2) $177: 34$
1995
(2) $145: 36$
(2) $163: 24$
(2) $177: 13$

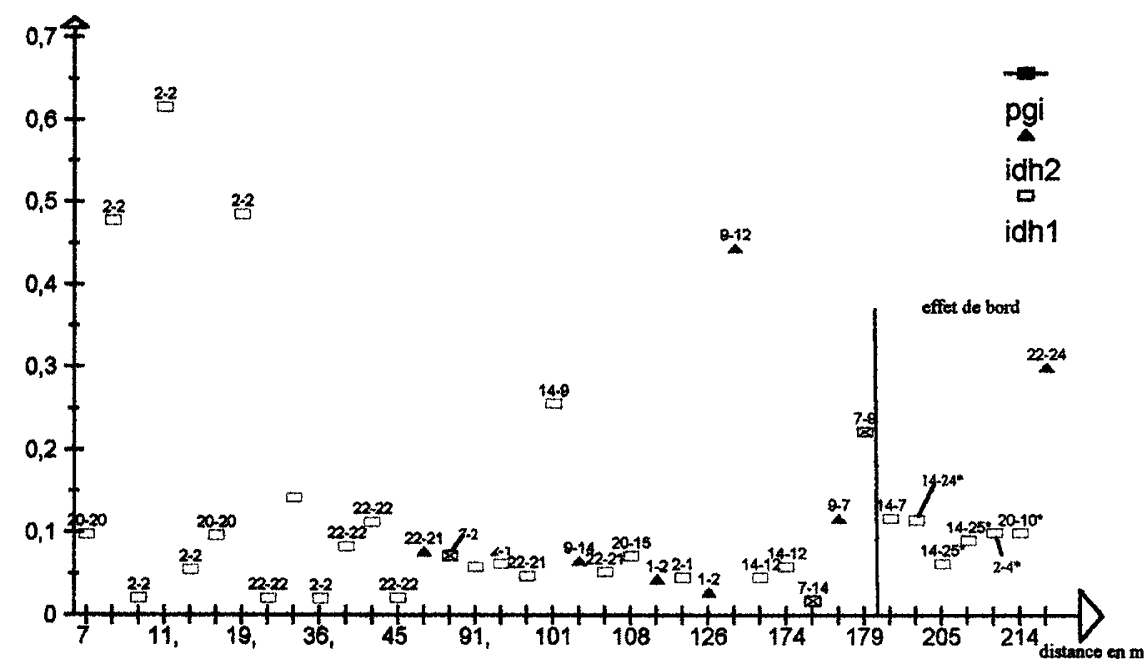

Figure 3. Distribution des allèles rares autour des pères (all 1 de pgi, all 3 de Idh1, all 2 de idh2) dans la P16. (le couple de nombres donne l'agrégat d'origine et l'agrégat receveur). 
spatiale agrégative ne semble pas favoriser les croisements entre apparentés, voisins proches dans le cas d'une faible distance de dissémination des graines. L'absence de relation entre taux moyen d'allofécondation de la population et densité de floraison, semble confirmer chez D. guianensis, une plus grande plasticité du système de reproduction par rapport aux facteurs environnementaux, comparativement à d'autres espèces d'arbres tropicaux (Murawski et Hamrick,1991).

La particularité de $D$. guianensis semble donc résider dans la grande stabilité du taux moyen d'allofécondation, tant à l'échelle spatiale (de 25 à $500 \mathrm{ha}$ ), que temporelle (entre deux années ayant des densités de floraison bien différentes). Elle serait en cela comparable à certains acacias, $A$. auriculiformis, $A$. crassicarpa, pour qui les facteurs environnementaux n'influencent que très faiblement le taux d'allofécondation, (Moran et al., 1989), et très différente d'autres espèces pollinisées par des animaux, comme Cavanillesia platanifolia (Murawski et Hamrick, 1992) et certains eucalyptus (Moran et Bell, 1983).

La plage de variation des valeurs de ti est comparable à celle observée chez $S$. oblongifolius $(0,4<t i<1)$, et est moins étendue que celle observée chez Ceiba pentandra et $C$. platnifolia $(0<t i<1)$, deux espèces pionnières des grandes trouées. En revanche, la moitié des arbres a, en moyenne, un taux d'allofécondation individuel significativement inférieur à 1 . Nous n'avons pas pu le corréler avec la densité de floraison du voisinage (tableau II).

La capacité de $D$. guianensis à accepter un certain pourcentage d'autofécondation, lui offre la possibilité de se maintenir et de se propager dans des conditions de densité très faible, et de coloniser des espaces libres au sein de la forêt, dans un environnement très compétitif ; ceci est à mettre en relation avec l'une des hypothèses de Marque (1996), qui pour expliquer la forte diversité intrapopulation de l'ADN chloroplastique, évoque pour cette espèce un fonctionnement en métapopulation, avec au sein d'une forêt, une mosaïque de phases de colonisation et d'extinction.

La quasi-stabilité interannuelle aux deux échelles spatiales du dispositif ( 500 ha) et de la parcelle 16 ( 25 ha) de la composition du nuage pollinique est remarquable. La bonne représentativité de nos échantillons ne suffit pas à expliquer ces résultats. La composition allèlique du nuage pollinique moyen au niveau de la population, reflète celle de la population de reproducteurs. Or, l'excès d'hétérozygotes constaté dans la population adulte $(F=-0,265)$ et l'absence apparente de structuration spatiale pour les marqueurs isoenzymatiques (Caron et al., en préparation), témoignent de la distribution assez uniforme des allèles les plus fréquents entre et à l'intérieur de chaque agrégat; en conséquence, la composition moyenne du nuage pollinique varie peu même si les arbres qui fleurissent diffèrent d'une année à l'autre.

Les allèles rares mettent en évidence que le rayon d'action des pollinisateurs est supérieur à la distance moyenne entre deux agrégats, ce qui leur permet de transporter du pollen entre des agrégats voisins. La réciprocité des échanges et leur répétabilité d'une année sur l'autre, montrent que la migration du pollen sur plusieurs centaines de mètres n'est pas un événement rare et que la valeur de $200 \mathrm{~m}$, est une estimation minimale, d'autant que l'échantillonnage n'étant pas suffisant, les effets de bords sont importants et que l'outil est par nature, peu discriminant. Cette valeur est très sensiblement inférieure à celle trouvée chez Tachigali versicolor et Platypodium elegans (Hamrick et Murawski, 1990). 
L'utilisation d'outils beaucoup plus performants, comme les marqueurs microsatellites, devrait permettre d'avoir une meilleure estimation de l'importance des flux polliniques à longues distances (Chase et al., 1996).

L'apparente contradiction d'une structuration spatiale en agrégat, forte pour les marqueurs chloroplastiques, et absente pour les isozymes, révèle une dissymétrie, en faveur de la voie mâle, des flux géniques par pollen et par graine. La possibilité de pollinisation à longue distance renforce les potentialités de l'espèce à s'installer dans des conditions de très faible densité.

Mais nos observations corroborent aussi, celles de plusieurs auteurs (Heinrich, 1979; Waddington, 1983) qui stipulent que les insectes pollinisateurs adaptent leurs déplacements à la disponibilité de la ressource : les échanges entre arbres les plus proches, à priori du même agrégat, semblent privilégiés, mais en fonction de la phénologie de l'année, le voisinage peut être constitué d'individus différents, ce qui peut expliquer les variations interannuelles de la composition moyenne du nuage pollinique de l'agrégat.

L'hétérogénéité des nuages polliniques individuels, est une constante de ce type d'étude. En plus de la phénologie des proches voisins, de l'abondance et de l'activité des pollinisateurs, des mécanismes d'incompatibilité, peuvent modifier significativement les fréquences alléliques et limiter le taux d'autofécondation.

Pour expliquer l'absence de modifications sensibles des taux de floraison et d'allofécondation du fait des traitements sylvicoles à Paracou, nous faisons l'hypothèse que l'importance des perturbations introduites par les traitements sylvicoles comparativement à celles des chablis, est insuffisante pour changer durablement l'écologie de la reproduction d'un arbre qui possède une plasticité suffisante pour se maintenir dans des conditions peu favorables. Il conviendrait de comparer une population témoin de forêt naturelle et celle d'une zone plus vaste et exploitée uniformément (plusieurs centaines d'hectares) avant de conclure sur cette question.

D. guianensis est l'une des huit espèces d'arbres étudiées dans notre laboratoire, pour lesquelles les études de la diversité génétique (niveau et structuration), et des flux géniques par graines (marqueurs chloroplastiques) sont en cours, basées sur les techniques ADN (RAPD, PCR-RFLP). La comparaison entre espèces ayant des attributs biologiques différents, devrait permettre de mieux comprendre l'influence de ces paramètres, en particulier du système de reproduction, sur la dynamique de la diversité génétique des arbres de la forêt tropicale humide de Guyane.

\section{REMERCIEMENTS}

Nos remerciements vont au Cirad-Forêt pour la mise à disposition du dispositif et de la base de données de Paracou, à A. Kremer pour la constance de ses conseils, à N. Haumont, B. Clément et T. Labbé pour leur aide précieuse dans l'utilisation des outils informatiques, à $\mathrm{H}$. Joly pour la relecture du manuscrit.

Cette étude a été financée dans le cadre d'un projet soutenu par le Bureau des ressources génétiques. 


\section{RÉFÉRENCES}

Bawa K.S., Plant pollinisator interactions in tropical rain forests, Annu. Rev. Ecol. Syst. 21 (1990) 399-422.

Bush R.M., Smouse P.E., Evidence for the adaptative significance of allozymes in forest trees, New Forest 6 (1992) 179-196.

Cabrera-Gaillard C., Gignoux J., Répartitions spatiales et sylviculture en forêt Guyanaise. Rapport interne Cirad, Kourou, 1989.

Chase M.R., Moller C., Kesseli R., Bawa K.S., Distant gene flow in tropical trees, Nature 383 (1996) 398-399.

Deschères P., Étude quantitative de la répartition spatiale de quelques espèces forestières commercialisables en Guyane Française, cas particulier : D. guianensis, Rapport de stage Engref, Kourou, 1991.

Doligez A., Joly H.I., Mating system of Carapa procera (Meliaceae) in the french Guiana tropical forest, Am. J. Bot. 84 (1997) 461-470.

Forget P.M., Dissémination et régénération naturelle de huit espèces d'arbres en forêt guyanaise, Thèse, Université Paris VI, 75-105, 1988.

Hamrick J.L., Godt M.J., Allozyme diversity in plant species, in : Brown A.H.D., CLegg M.T., Kahler A.L., Weir B.S., (Eds), Population genetics and germ-plasm resources in crop improvement, Sinauer, Sunderland, MA, 44-64, 1989.

Hamrick J.L., Loveless M.D., The genetic structure of tropical tree populations : association with reproductive biology, in: Bock J.H., Linhart Y.B., (Eds), The evolutionary ecology of plants, Westview Press, Boulder, CO, 129-146, 1989.

Hamrick J.L., Murawski D.A., The breeding structure of tropical tree populations, Plant Species Biol. 5 (1990) 157-165.

Heinrich B., Ressource heterogeneity and patterns of movement in foraging bumble bees, Oecologia 40 (1979) 235-246.

Liengsiri C., Piewlang C., Boyle T.J.B., A manual of starch gel electrophoresis of tropical trees. Asean Canada Forest Tree Seed Centre, Thailland, 1990.

Loubry D., Les paradoxes de l'angélique (Dicorynia guianensis Amshoff) : dissémination et parasitisme des graines avant dispersion chez un arbre anémochore de la forêt guyanaise, Rev. Ecol. (Terre et Vie) 48 (1993) 353-363.

Loubry D., Déterminisme du comportement phénologique des arbres en forêt tropicale humide de Guyane française ( $5^{\circ}$ lat. N.), thèse, Université Paris VI, 104-123, 1994.

Marque G., Variabilité intra- et interpopulations de l'ADN chloroplastique chez l'angélique de Guyane (Dicorynia guianensis), Mémoire de DEA de biologie forestière, Université de Nancy 1, 1996.

Moran G.F., Bell J.C., Eucalypts, in : Tanksley S.D., Orton T.J. (Eds) Isozymes in plant genetics and breeding, Elsevier Science Publishers, Amsterdam, part B, 43-431, 1983.

Moran G.F., Muona O., Bell J.C., Breeding systems and genetic diversity in Acacia auriculiformis and A. crassicarpa, Biotropica 21 (1989) 250-256.

Murawski D.A., Hamrick J.L., The effect of the density of flowering individuals on the mating systems of nine tropical tree species, Heredity 67 (1991) 167-174.

Murawski D.A., Hamrick J.L., Mating system and phenology of Ceiba pentandra (Bombacaceae) in central Panama, J. Hered. 83 (1992a) 401-404.

Murawski D.A., Hamrick J.L., The mating system of Cavanillesia platanifolia under extremes of flowering-tree density: a test of predictions, Biotropica 24 (1992b) 99-101.

Murawski D.A., Gunatilleke N., Bawa K.S., The effects of selective logging on inbreeding in Shorea megistophylla (Dipterocarpaceae) from Sri Lanka, Conservation Biology 8 (1994) 997-1002. 
Murawski D.A., Reproductive biology and genetics of tropical trees from a canopy perspective, in: Loman M., Nadkarni N. (Eds), Forest Canopies, Academic Press, N.Y., 457-493, (1995).

Ritland K., A serie of FORTRAN computer programs for estimating plant mating system, J. Hered. 81 (1990) 235-237.

Ritland K., Jain S.K., A model for the estimation of outcrosing rate and gene frequencies based on $\mathrm{n}$ independent loci, Heredity 47 (1981) 37-54.

Ritland K., El-Kassaby Y.A., The nature of inbreeding in a seed orchard of Douglas fir as shown by an efficient multilocus model, Theor. Appl. Genet. 71 (1985) 375-384.

Schmitt L., Étude des peuplements naturels en forêt dense guyanaise. Compte rendu de mise en exploitation forestière du dispositif sylvicole de Paracou, CTFT, Kourou, 1989.

Waddington K.D., Foraging behavior of pollinators, in : Real L.A. (Ed), Pollination Biology, Academic press, Orlando, 213-239, 1983. 